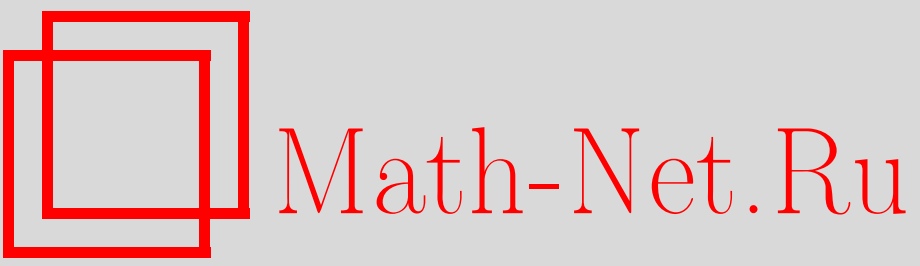

Д. С. Бойков, А. С. Болдарев, В. А. Гасилов, Численный анализ магнитомеханического эффекта в нагревательных трубах, Матем. моделирование, 2020, том 32 , номер 4, 3-15

DOI: https://doi.org/10.20948/mm-2020-04-01

Использование Общероссийского математического портала Math-Net.Ru подразумевает, что вы прочитали и согласны с пользовательским соглашением

http: //www . mathnet.ru/rus/agreement

Параметры загрузки:

IP : 107.22 .136 .117

26 апреля 2023 г., 14:02:41 


\title{
ЧИСЛЕННЫЙ АНАЛИЗ МАГНИТОМЕХАНИЧЕСКОГО ЭФФЕКТА В НАГРЕВАТЕЛЬНЫХ ТРУБАХ
}

\author{
(C) 2020 г. Д.С. Бойков, А.С. Болдарев, В.А. Гасилов \\ ИПМ им. М.В. Келдыша РАН, Москва gasilov@keldysh.ru
}

DOI: $10.20948 / \mathrm{mm}-2020-04-01$

Рассмотрена физико-математическая модель, позволяющая исследовать перспективы использования эффекта «магнитной памяти металла» с целью неразрушающего контроля изделий из ферромагнитных материалов, находящихся в магнитном поле Земли. На основе метода конечных элементов разработаны алгоритм и программа для расчета в трехмерной постановке задачи о распределении магнитного потенциала в среде с неоднородной магнитной проницаемостью. Созданные методики и программные средства использованы для моделирования поля рассеяния около защемленных нагревательных труб паровых котлов. Получены соотношения, показывающие взаимосвязь между изменением магнитной индукции и магнитным полем рассеяния. Отмечено удовлетворительное совпадение расчетных и опытных данных распределения напряженности магнитного поля рассеяния на поверхности нагревательной трубы.

Ключевые слова: ферромагнетизм, «магнитная память металла», неразрушающий контроль нагревательных труб, математическое моделирование.

\section{NUMERICAL ANALYSIS OF THE MAGNETOMECHANICAL EFFECT IN HEATING PIPELINES}

\section{D.S. Boykov, A.S. Boldarev, V.A. Gasilov}

Keldysh Institute of Applied Mathematics, Russian Ac. Sci., Moscow

We considered a physico-mathematical model which allows to explore the possibilities of the utilization of the «magnetic memory of metals» effect with the aim of nondestructive testing of the items made of ferromagnetic materials placed into the Earth's magnetic field. Based on the finite elements method, an algorithm and a computer code for the 3D computations of the magnetic potential distribution in a medium with nonuniform magnetic permeability were developed. The developed methods and software tools were used for the modelling of the leakage field near the fixed-ended heating pipes of the steam boilers. Relationships demonstrating the interrelation between the magnetic induction change and the magnetic leakage field were obtained. A satisfactory coincidence of computational and experimental data concerning the intensity of the magnetic leakage field distribution on the surface of the heating tube was noted. 
Keywords: ferromagnetism, «magnetic memory of metal», nondestructive testing of heat pipelines, mathematical modelling.

\section{1. Введение}

Перегрев металлоконструкций в зоне интенсивного нагрева, появляющийся при нештатных режимах эксплуатации энергетического оборудования может приводить к появлению зон концентраций термомеханических напряжений. Подобные ситуации имеют место при перегревах котельных труб, которые в силу конструкционных условий должны быть жестко закреплены («защемлены») в определенных местах. Эти места и оказываются концентраторами термомеханических напряжений. Таким образом, на отдельных участках труб уровень напряжений оказывается значительно выше допустимого, вызывая тем самым пластическую деформацию. На данных участках труб процессы коррозии, ползучести и появления дефектов (усталость металла) протекают наиболее интенсивно. Для своевременного выявления участков труб, предрасположенных к повреждениям, и снижения ремонтных затрат, разрабатываются неразрушающие методы контроля, имеющие корреляцию с механическими напряжениями.

Среди известных диагностических методов (оптических, акустических, радиационных, магнитных) наиболее перспективными для оценки механических напряжений представляются магнитные методы. Широко применяется метод, основанный на магнитной памяти металла [1], проявляющейся в возникновении локальной магнитной аномалии в областях концентрации напряжений и деформаций.

В силу магнитомеханического эффекта магнитное поле рассеяния соответствующего направления и, как правило, со сменой знака наблюдается в том случае, когда обладающее ферромагнитными свойствами тело (например, конструкционный элемент из стали, обладающей ферромагнитными свойствами) подвергается тепловым или механическим воздействиям, приводящим к большим локальным нарушениям однородности магнитных свойств. Как известно, магнитное поле в определенном температурном диапазоне и в отсутствие значительных механических напряжений усиливается внутри ферромагнетика, благодаря чему его силовые линии имеют характерный вид «пучка, втянутого внутрь тела». Если магнитная проницаемость по какой-либо причине в каком-либо месте существенно уменьшается, то в окрестности этой зоны падения проницаемости магнитное поле весьма неоднородно, а силовые линии поля «выпучены» в окружающее пространство. Об этом эффекте часто говорят как о появлении «поля рассеяния».

Основными достоинствами данного метода являются: 
- использование данного метода диагностики не требует применения специальных намагничивающих устройств, поскольку использует явление намагничивания узлов и агрегатов в процессе работы в магнитном поле Земли;

- не требуется специальной подготовки контролируемой поверхности;

- позволяет выполнять раннюю диагностику оборудования, на основе проведенных измерений и уже известных экспериментальных данных можно сделать прогноз о надежности испытуемого оборудования.

Данный метод позволяет производить оценку состояния контролируемых объектов с учетом материала, условий эксплуатации, особенностей элементов конструкций. Зоны концентрации деформаций при использовании этого метода предложено фиксировать на поверхности труб по магнитным полям рассеяния (МПР).

Зависимость между магнитным состоянием тел и механическими нагрузками описывается в теории ферромагнетизма $[2,3]$ как один из частных случаев общей параметрической зависимости намагниченности ферромагнетиков от температуры, напряженности и направления внешнего магнитного поля, внутренних механических напряжений, химического состава металлов. Из результатов экспериментальных исследований [4] следует, что у ферромагнетика, находящегося в магнитном поле под действием механических нагрузок возникает анизотропия магнитных свойств, т.е. магнитная проницаемость среды становится тензорной величиной. Вследствие такой сложной зависимости магнитных свойств от многих параметров анализ результатов измерений МПР для котельных труб становится во многих случаях весьма затруднительным. В связи с этим наиболее актуальной становится разработка математической модели намагничивания, которая позволила бы на основе численного анализа выделить роль отдельных конструктивных и эксплуатационных факторов.

Анализ результатов экспериментальных и промышленных исследований $[1,4-6]$ позволяет построить математическую модель распределения МПР на поверхности трубы при одновременном воздействии механических нагрузок (от внутреннего давления и температурного расширения) и внешнего поля Земли. Распределение температуры в трубе считается известным, а механические напряжения рассчитываются для каждого конкретного случая.

\section{2. Методика проведения расчета}

При отсутствии токов проводимости стационарное магнитное поле в среде описывается уравнениями Максвелла.

$\operatorname{Rot} \mathbf{H}=0, \quad \operatorname{div} \mathbf{B}=0$,

где $\mathbf{B}$ - магнитная индукция; $\mathbf{H}$ - напряженность магнитного поля; $\mathbf{B}=$ 
$=\mathbf{H}+4 \pi \mathbf{J} ; \mathbf{J}-$ вектор намагниченности материала [7]. Из (1) следует, что $\mathbf{H}$ может быть представлена в виде градиента некоторой скалярной функции потенциала магнитостатического поля $\mathbf{H}=\operatorname{grad} \varphi$. Если принять, что вектор магнитной индукции пропорционален вектору напряженности магнитного поля $\mathbf{H}$, то, вводя коэффициент магнитной проницаемости $\mu(\mathbf{B}=\mu \mathbf{H})$, уравнение для магнитного потенциала можно представить в виде

$$
\operatorname{div}(\mu \operatorname{grad} \varphi)=0 .
$$

Отметим, что согласно данным [2,3] величина $\mu$ в случае анизотропии магнитных свойств материала является тензором. Следовательно, в рамках данной модели ставится следующая задача магнитостатики: найти скалярный потенциал, удовлетворяющий уравнению (2) с переменными (вообще говоря, разрывными) коэффициентами, зависящими от напряженности магнитного поля, температуры и механических напряжений.

В качестве граничного условия на бесконечности напряженность магнитного поля считается равной напряженности невозмущенного поля Земли. Распределение температуры в трубе считается известным, а механические напряжения рассчитываются для каждого конкретного случая.

Решение задачи о распределении МПР включает три основных этапа:

- расчет механических напряжений в защемленной трубе, потерявшей устойчивость в результате температурного расширения;

- расчет магнитной проницаемости с использованием полученных напряжений;

- решение уравнения для магнитного потенциала поля рассеяния и вычисление МПР на поверхности трубы.

Для расчета магнитной проницаемости $\mu$ важно выделить участки с пластическими и упругими деформациями, так как по мере увеличения температурного расширения в трубе, не имеющей температурной компенсации, отдельные участки могут перейти в пластическое состояние. В реальных условиях работы котла в трубах часто возникают остаточные деформации типа изгиба с кручением. Расчет напряжений в трубах, находящихся в упругопластическом состоянии, представляется достаточно сложной самостоятельной задачей. В данной работе задача точного определения механических напряжений и деформаций с учетом пластичности не ставилась. Рассматривается приближенная модель механического состояния с условием, что модуль упругости во всём диапазоне напряжений есть величина постоянная. Для такой модели важным является выбор такой зависимости $\mu$ от механических напряжений, которая бы отражала особенности изменения 
магнитных свойств по мере увеличения напряжений растяжения и сжатия, в частности, при переходе от упругих к пластическим деформациям. Для этого использовались известные опытные данные по магнитным свойствам малоуглеродистых сталей в слабых магнитных полях $\mathbf{H} \leq 50 \mathrm{~A} / \mathrm{M}$ :

при растяжении необратимая составляющая магнитной индукции увеличивается в 5 раз и более по сравнению с индукцией в ненагруженном состоянии. При сжатии необратимая составляющая в 2-3 раза меньше, чем при растяжении [1];

при увеличении растягивающих напряжений на материалы с положительной магнитострикцией магнитная проницаемость возрастает до достижения предела упругости, после чего проницаемость монотонно снижается $[2,3]$;

зависимость магнитной проницаемости от внешнего поля имеет линейный характер [3].

Кроме того, учитывались особенности намагничивания в полях, ориентированных перпендикулярно направлению растягивающих и сжимающих напряжений $[8,9]$.

Напряженность магнитного поля Н вокруг ферромагнитного тела на бесконечном расстоянии от него принимается равной напряженности невозмущенного магнитного поля Земли. Распределение температуры $T$ в теле считается заданным, а механические напряжения $\sigma$ или задаются, или рассчитываются согласно условиям задачи.

В настоящей работе функциональная зависимость магнитной проницаемости в виде $\mu=f(\sigma, H, T)$ принята с использованием экспериментальных данных по одноосному нагружению образцов. Для случая слабых магнитных полей

$$
\mu=\mu_{0}\left(1+\frac{b}{\mu_{0}} H\right) G(\sigma), \quad b=\text { const }, \quad \mu=f(T) .
$$

Для стали 20 в диапазоне температур $20-700^{\circ} \mathrm{C}$, т.е. ниже точки Кюри, используется зависимость, предложенная в работе [5]:

$$
\mu_{0}(T)=285.58-0.99791 \cdot 10^{-1} T+0.99676 \cdot 10^{-2} T^{2}-0.42674 \cdot 10^{-5} T^{3} .
$$

Величина $b=2.5$ принята из обобщенных опытных данных [8] для горячекатаной стали 20.

Зависимость $G(\sigma)$ представлена в виде

$K(\sigma)=A+B|\sigma|^{m} \exp (n|\sigma|)$. 
При сжатии $(\sigma<0)$ возможны случаи

$|\sigma|<50$ МПа: $A=B=1.0 ; \quad m=1 ; \quad n=-2 ;$

$50 \mathrm{M \Pi а} \leq|\sigma| \leq 250$ МПа: $A=28.0 ; \quad B=0 ;$

$250 \mathrm{M \Pi а}<|\sigma|: \quad A=0.0 ; \quad B=1.691 \cdot 10^{-6} ; \quad m=6.46$.

При растяжении $(\sigma>0)$ :

$\sigma<50$ МПа: $A=0.0 ; \quad B=1.182 ; \quad m=2 ; \quad n=-0.4$

$\sigma \geq 50$ МПа: $A=0.0 ; \quad B=2.718 ; \quad m=1 ; \quad n=-0.2$.

В модели учитываются особенности намагничивания в полях, ориентированных перпендикулярно направлению растягивающих и сжимающих напряжений [9]. Согласно [9] прирост намагниченности в магнитном поле $\mathbf{H} \perp \mathbf{F}$ (F-Механическая сила) равен ее приросту в случае $\mathbf{H}|| \mathbf{F}$ при условии, что коллинеарное напряжение имеет знак, обратный знаку действующего напряжения, а его значение равно этому напряжению, умноженному на коэффициент Пуассона. Поэтому для рассматриваемой постановки задачи принят упрощенный (диагональный) вид тензора магнитной проницаемости, в котором исходя из формулы (3) учтены только диагональные компоненты $\sigma$, соответствующие направлениям осей $x, y, z: \mu_{i}=f\left(\sigma_{i}, H_{i}, T\right)$, где $i=1,2,3$.

Необходимо отметить, что использованная в расчетах зависимость $\mu$ имеет качественный характер и может рассматриваться лишь как функция первого приближения. По мере накопления опытных данных по магнитным свойствам для сложных условий одновременного действия высоких температур и механических напряжений зависимость (3) будет уточнена.

Комплекс расчетных программ, разработанный на основе метода конечных разностей [10], включает блоки решения задач теплопроводности, упругости с учетом переменных по пространству параметров упругости как следствие неоднородного теплового поля, а также задач определения потенциала поля рассеяния. Комплекс программ создан с использованием инфраструктуры MARPLE [11]. Расчет выполнялся в переменных Лагранжа. Состояние деформируемого материала описывается с помощью модели Прандтля-Рейса с учетом релаксации касательных напряжений в варианте, предложенном в [12]. Для замыкания системы уравнений используется уравнение состояния в форме Ми - Грюнайзена.

\section{3. Результаты расчетов}

Задача 1. Рассмотрена задача о расчете поля рассеяния для неоднородно нагруженной ферромагнитной трубы, помещенной в среду с «вакуумной» магнитной проницаемостью. Геометрическая область, в которой ре- 
шалась задача, представлена на рис. 1 , где $a=0.03 ; b=0.024$ соответственно внешний и внутренний радиусы трубы.

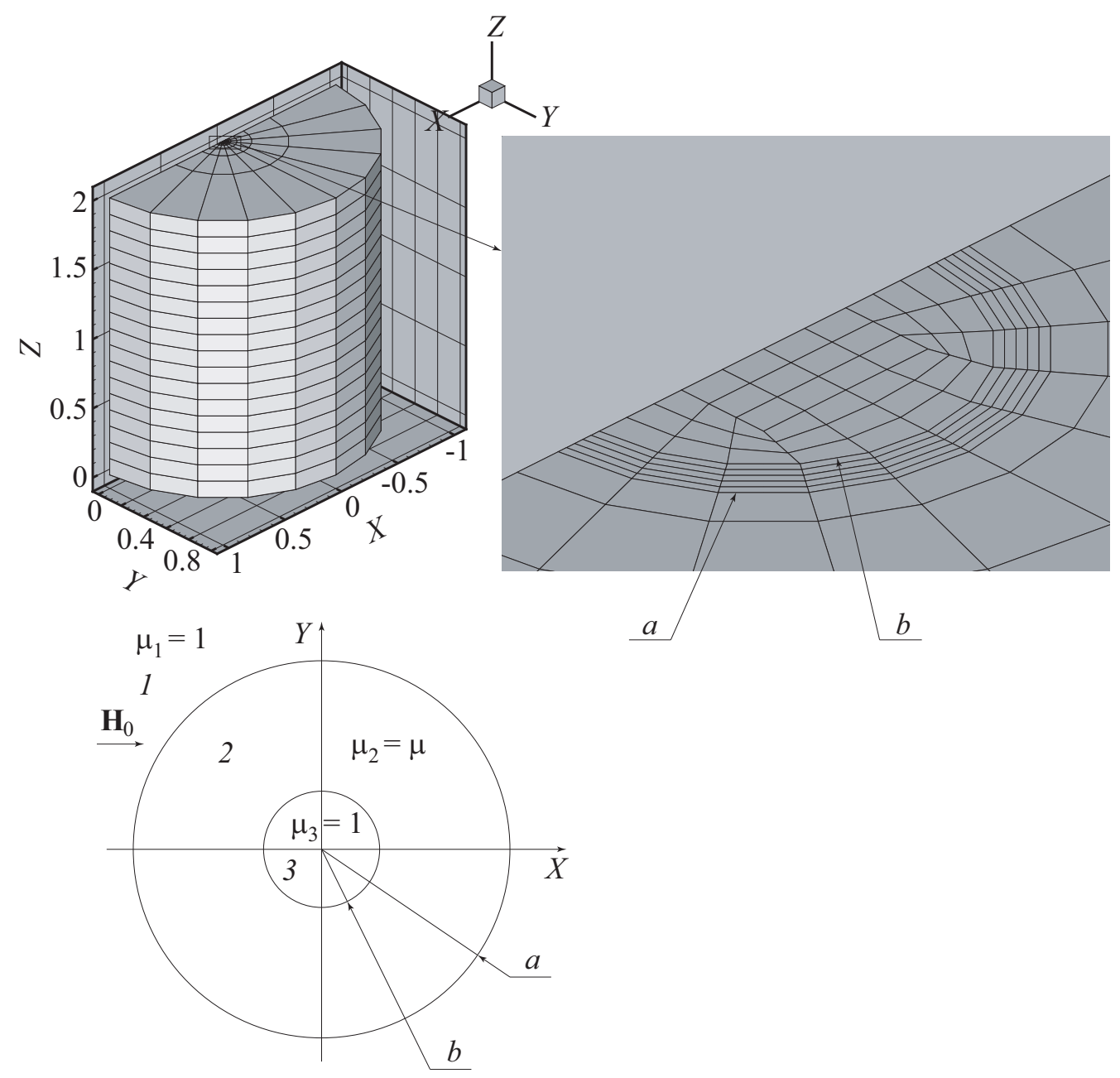

Рис.1.

Невозмущенное однородное магнитное поле величины $H_{0}=10.0$ направлено перпендикулярно оси трубы. В расчете предполагалось, что ось недеформированной трубы совпадает с направлением оси $z$. Предполагается, что в координатной плоскости $(x-y)$ лежит горизонтальная составляющая поля Земли.

Первоначально было рассчитано магнитное поле в случае постоянной магнитной проницаемости трубы, равной $\mu=100$. В указанной постановке задачи распределение магнитного поля может быть получено аналитически методами, изложенными в [2, 3]:

$$
\begin{aligned}
& \lambda=b^{2} / a^{2}, \\
& H_{1 x}=H_{0}\left(1-a^{2} \frac{\left(\mu^{2}-1\right)(1-\lambda)}{(\mu+1)^{2}-(\mu-1)^{2} \lambda} \cdot \frac{y^{2}-x^{2}}{\left(y^{2}+x^{2}\right)^{2}}\right),
\end{aligned}
$$




$$
\begin{aligned}
& H_{1 y}=H_{0} a^{2} \frac{\left(\mu^{2}-1\right)(1-\lambda)}{(\mu+1)^{2}-(\mu-1)^{2} \lambda} \cdot \frac{2 x y}{\left(y^{2}+x^{2}\right)^{2}}, \\
& H_{2 x}=2 H_{0}\left(\frac{\mu+1}{(\mu+1)^{2}-(\mu-1)^{2} \lambda}+b^{2} \frac{\mu-1}{(\mu+1)^{2}-(\mu-1)^{2} \lambda} \cdot \frac{y^{2}-x^{2}}{\left(y^{2}+x^{2}\right)^{2}}\right), \\
& H_{2 y}=2 H_{0} b^{2} \frac{\mu-1}{(\mu+1)^{2}-(\mu-1)^{2} \lambda} \cdot \frac{2 y x}{\left(y^{2}+x^{2}\right)^{2}}, \\
& H_{3 x}=H_{0}\left(\frac{4 \mu}{(\mu+1)^{2}-(\mu-1)^{2} \lambda}\right), \quad H_{3 y}=0 .
\end{aligned}
$$

Индексы 1, 2 и 3 соответствуют областям, помеченным как 1,2 и 3 на рис.1.

Решение, представленное формулами (6) было использовано для верификации программного комплекса. В таблице представлены результаты численного моделирования в норме $L_{2}$ :

\begin{tabular}{|c|c|}
\hline$\frac{1}{\sqrt{V}} \int_{V}\left(H_{x}\right)^{2} d V=9.975637$ & $\frac{1}{\sqrt{V}} \int_{V}\left(H_{y}\right)^{2} d V=7.417031 \cdot 10^{-1}$ \\
\hline$\frac{1}{\sqrt{V}} \int_{V}\left(H_{x}-\frac{\partial \varphi}{\partial x}\right)^{2} d V=9.832729 \cdot 10^{-3}$ & $\frac{1}{\sqrt{V}} \int_{V}\left(H_{y}-\frac{\partial \varphi}{\partial y}\right)^{2} d V=2.32861 \cdot 10^{-3}$ \\
\hline
\end{tabular}

Задача 2. Рассмотрена задача о расчете поля рассеяния для неоднородно нагруженной ферромагнитной трубы, помещенной в среду с «вакуумной» магнитной проницаемостью для случая неоднородного распределения коэффициента магнитной проницаемости (расчетная область - см. задача 1).

В рассматриваемом примере предполагалось, что в области $(0.75 \leq Z \leq 1.25$; $-0.015 \leq Y \leq 0.015 ;-0.015 \leq X \leq 0.015)$ имеет место пластическая деформация $(\mu=3)$. В остальной области магнитная проницаемость трубы принималась равной $\mu=100$. На рис. 2 приведены результаты численного моделирования.

Задача 3. Исследована задача о МПР, возникающих в защемленной вертикально расположенной стальной трубе. Невозмущенное внешнее поле соответствует географической широте московского региона: $H_{x}=10 \mathrm{~A} / \mathrm{M}, H_{y}=$ $=-40 \mathrm{~A} / \mathrm{M},(x, y, z)$ - декартова система координат, использовавшаяся в расчетах. Ось недеформированной трубы совпадает с направлением оси $Z$. Предполагается, что в координатной плоскости $(x-z)$ лежат горизонтальная 
составляющая поля Земли и стрела прогиба трубы, в силу чего это будет одновременно и плоскость симметрии задачи. Расчеты проводились для температурных режимов, при которых труба теряет механическую устойчивость.

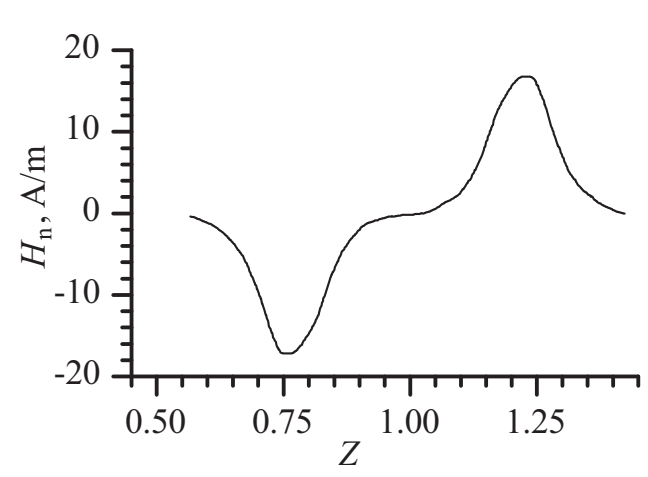

Pис.2.

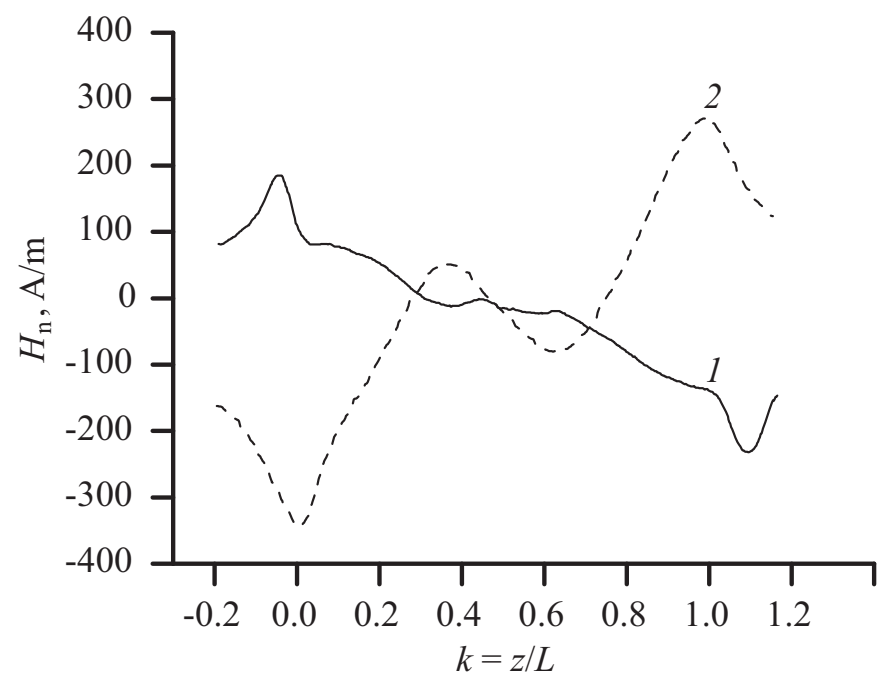

Рис.3. Изменение нормальной составляющей магнитного поля рассеяния вдоль оси на наружной поверхности вертикальной трубы, защемленной в точках $k=0$ и $k=1$. $\Theta=0$, $D=0.06 \mathrm{м}, d=0.048 \mathrm{м}, T=350^{\circ} \mathrm{C}, p=15 \mathrm{M}$ Па. 1: $L=4 \mathrm{M}, \Delta T=70^{\circ} \mathrm{C} ; 2: L=2 \mathrm{M}, \Delta T=230{ }^{\circ} \mathrm{C}$.

Результаты расчетов приведены для двух значений расстояния между точками защемления трубы: $L=2$ и 4 м и соответственно для двух значений температуры перегрева трубы в тепловом экране: 230 и $70^{\circ} \mathrm{C}$. В обоих случаях возникает потеря устойчивости трубы, однако при различных значениях механических напряжений, а именно в отношении

$$
\sigma_{1 \kappa p}: \sigma_{2 \kappa p}=1: 2 .
$$

На рис.3 приведено распределение вдоль оси $Z$ (в зависимости от $k=$ $=z(L)$ нормальной к поверхности трубы компоненты МПР $-H_{x}$ при $\Theta=0$. Характер распределения напряженности магнитного поля для обоих случаев различен. Для более длинной трубы ( $L=4$ м, линия 1$)$ характерно монотонное повышение $H_{x}$ в направлении поля Земли, т.е. от верхней точки защемления трубы $(k=1)$ до нижней $(k=0)$ : от -215 до $185 \mathrm{~A} / \mathrm{M}$. Для короткой трубы ( $L=2$ м, линия 2) наоборот, напряженность магнитного поля $H_{\mathrm{x}}$ понижается от 270 до $-320 \mathrm{~A} / \mathrm{M}$. Распределение для короткой трубы немонотонное - имеются максимум $H_{x}=51 \mathrm{~A} /$ м и минимум $H_{x}=-80 \mathrm{~A} /$ м в точках $k=0.35$ и $k=0.65$. Такое распределение МПР обусловлено изменением магнитной проницаемости в металле трубы как функции от механических напряжений.

На рис.4 в координатах $(z-\Theta)$ показано распределение магнитной проницаемости $\mu(z)$ на развертке стенки трубы, длина которой $L=2$ м. Ввиду 
указанной симметрии распределение дано в пределах $0 \leq \Theta \leq \pi$. Показаны изолинии магнитной проницаемости в диапазоне $2 \leq \mu(z) \leq 1300$. Области, охватываемые изолиниями $\mu(z)=2$, можно интерпретировать как области пластической деформации, где согласно критерию Мизеса [13] в приближении тонкостенной трубы имеем

$$
\sigma^{2} 0.5\left(\sigma_{z z}^{2}+\sigma_{\Theta \Theta}^{2}+\left(\sigma_{z z}^{2}+\sigma_{\Theta \Theta}^{2}\right)\right)+3 \sigma_{\Theta z}^{2}+\sigma_{T}^{2} .
$$

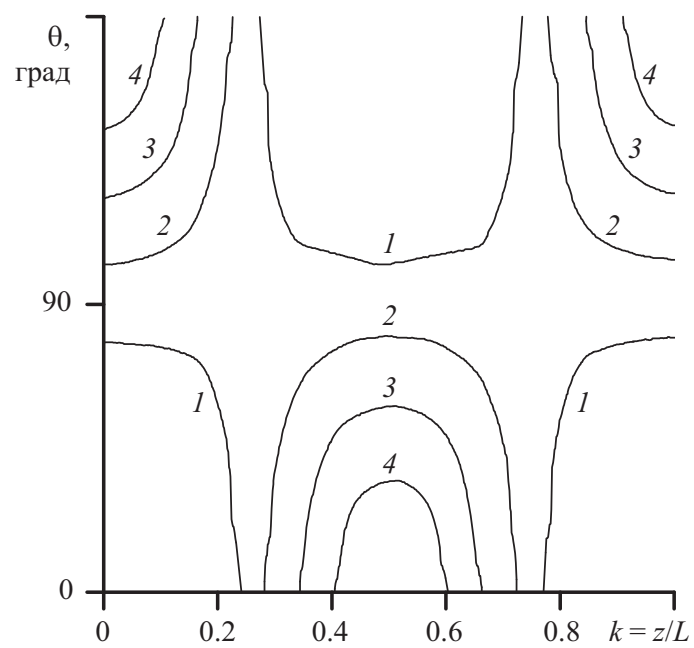

Рис.4. Распределение магнитной проницаемости на развертке трубы. Изолинии магнитной проницаемости, $\mu(z): 1-2 ; 2-30 ; 3-300 ; 4-1300 ; L=2$ м. Остальные параметры на рис.1.

Перераспределение магнитной проницаемости соответственно полю механических напряжений приводит к перераспределению магнитного потока в теле трубы и образованию МПР. Распределение нормальной к поверхности трубы компоненты МПР $H_{x}$ вдоль оси трубы при $\Theta=0$ показано на рис.3. Отметим соответствие распределений $H_{x}$ и магнитной проницаемости $\mu(z)$. В силу анизотропии значения $\mu(z), \mu(\Theta), \mu(r)$ не равны между собой. Образование в каком-либо месте трубы участка пластической деформации и соответственно значительное уменьшение $\mu(z)$ проявляется в виде возникновения поля рассеяния по всему периметру трубы вне зависимости от локализации этого участка по азимуту. При перемещении в направлении вектора внешнего магнитного поля распределение МПР носит «волнообразный» характер, т.е. появляются несколько точек локальных экстремумов - вблизи точек защемления и между ними. Знак $H_{x}$ в точке экстремума зависит от знака приращения магнитного сопротивления материала $\Delta R_{m}, R_{m}==1 / \mu(z)$, при движении вдоль данного направления: если $\Delta R_{m}>0$, то $H_{n}<0$ в верхней точке и $H_{n}>0$ в нижней точке (линия 1, рис.3). Знаки экстремумов $H_{n}$ меняются на противоположные при $\Delta R_{m}<0$ (линия 2, рис.4). Амплитуда МПР пропорциональна величине приращения $\Delta R_{m}$, протяженности участка с аномалией магнитных свойств и напряженности внешнего поля. 
Таким образом, появление экстремумов при распределении МПР означает существование областей с аномальным магнитным сопротивлением. Поскольку $\mu=f(\sigma, H, T)$, то анализ измеренных распределений МПР позволяет получить информацию о состоянии металла и воздействии на него механических и тепловых нагрузок. Такая информация, полученная в процессе промышленных и лабораторных исследований, была положена в основу метода диагностики состояния труб с использованием магнитной памяти металла [1].

\section{4. Выводы}

1. Для котельных труб, эксплуатируемых в условиях недостатка температурной компенсации, распределение нормальной составляющей магнитного поля рассеяния на поверхности котельных труб, измеренной в ходе промышленных исследований, соответствует рассчитанной по предложенной математической модели.

2. Возникновение магнитных полей рассеяния (МПР) на поверхности магнитозамкнутых котельных труб обусловлено в основном недостатком температурной компенсации при тепловом расширении. МПР возникают в местах локальных неоднородностей магнитной проницаемости и соответственно неоднородностей механических напряжений. Знак $H_{x}-$ нормальной к поверхности трубы компоненты МПР - зависит от изменения магнитного сопротивления в направлении вектора магнитного поля Земли. Место смены знака $H_{x}$ совпадает с границей между областями упругой и пластической деформаций материала трубы, т.е. по расположению изолиний $H_{x}=0$ можно выделить зоны упругих и пластических деформаций. В реальных условиях смена знака $H_{x}$ может быть обусловлена расположением дефектов металла и мест концентрации напряжений и деформаций.

3. Предложенная в работе модель намагничивания и созданное на ее основе программное обеспечение дают возможность производить расчеты МПР для различных схем нагружения, температурно-неоднородных режимов, в том числе сопровождающихся появлением зон пластических деформаций. Это позволит в дальнейшем по измеренным полям $H_{x}$ решать задачу количественной оценки нагрузок на котельные трубы, вызванных недостатком самокомпенсации и другими нерасчетными условиями.

\section{СПИСОК ЛИТЕРАТУРЫ}

1. Н.Н. Зацепин, Л.Б. Коржова. Магнитная дефектоскопия. - Минск: Наука и техника, 1981, 208c.;

N.N.Zatsepin, L.B. Korzhova. Magnitnaia defektoskopiia. - Minsk: Nauka i tekhnika, 1981, $208 \mathrm{~s}$. 
2. С.В. Вонсовский, Я.С. Шур. Ферромагнетизм. - М.: ОГИЗ. Гос. изд. техн.- теор. литры, 1948.

S.V. Vonsovskii, Ia.S. Shur. Ferromagnetizm. - M.: OGIZ. Gos. izd. tekhn.-teor. lit-ry, 1948.

3. Р. Бозорт. Ферромагнетизм. - М.: Изд. иностран. лит-ры, 1956.

R. Bozort. Ferromagnetizm. - M.: Izd. inostran. lit-ry, 1956.

4. Э.С. Горкунов, В.Ф. Новиков, А.П. Ничипурук, В.В. Насонов, А.В. Кадров, И.Н. Татлыбаева. Устойчивость остаточной намагниченности термически обработанных стальных изделий к действию упругих деформаций // Дефектоскопия, 1991, №2, с.68-76;

E.S. Gorkunov, V.F. Novikov, A.P. Nichipuruk, V.V. Nassonov, A.V. Kadrov, I.N. Tatlybaeva. Resistance of residual magnetization of heat-treated steel products to elastic deformations // Soviet Journal of Nondestructive Testing, 1991, v.27, №2, p.138-145.

5. В.А. Богачёв, М.И. Гончар, Е.И. Дарвин. Магнитный метод определения перегретых труб из сталей 20 и 12Х1МФ поверхностей нагрева паровых котлов // Электрические станции, 1995, № 3, с.9-13;

V.A. Bogachev, M.I. Gonchar, E.I. Darvin. Magnitnyi metod opredeleniia peregretukh trub iz stalei 20 i 12KH1MF poverkhnostei nagreva parovykh kotlov // Elektricheskiie stantsii, 1995, №3, s.9-13.

6. А.А. Дубов. Диагностика котельных труб с использованием магнитной памяти металла. - М.-Л.: Энергоиздат, 1995.

A.A. Dubov. Diagnostika kotelnykh trub $\mathrm{s}$ ispolzovaniem magnitnoi pamiati metalla. M.-L.: Energoizdat, 1995.

7. Л.Д. Ландау, Е.М. Лифииц. Электродинамика сплошных сред. - М.: Физматлит, 2001, 656c.;

L.D. Landau, E.M. Lifshitz, L.P. Pitaevskii. Electrodynamics of Continuous Media. - Butterworth-Heinemann, 1984.

8. А.А. Преображенский. Теория магнетизма, магнитные материалы и элементы. - М.: Высшая школа, 1972;

A.A. Preobrazhenskii. Teoriia magnetizma, magnitnyie materialy i elementy. - M.: Vysshaia shkola, 1972.

9. В.Г. Кулеев, М.Б. Ригмант. Особенности магнитоупругих явлений в ферромагнитных сталях в малых магнитных полях, перпендикулярных направлению действия циклических растягивающих и сжимающих напряжений // ФММ, 1995, т. 79, №1, с.120-129;

V.G. Kuleev, M.B. Rigmant. Osobennosti magnitouprugikh iavlenii v ferromagnitnykh staliah v malykh magnitnykh poliah, perpendikuliarnykh napravleniiu deistviia tsiklicheskikh rastiagivaiushchikh i szhimaiushchikh napriazhenii // FMM, 1995, v.79, №1, s.120-129.

10. М.Л. Уилкинс, С. Френч, М. Сорем. Конечно-разностная схема для решения задач, зависящих от трех пространственных координат и времени // Численные методы в механике жидкостей. - М.: Мир, 1973, с. 115-119;

M.L. Wilkins, S.J. French, M. Sorem. Finite difference scheme for calculating problems in three space dimensions and time // Proceedings of the Second International Conference on Numerical Methods in Fluid Dynamics. - New York: 1971, p.30-33.

11. В.А. Гасилов, Г.А. Багдасаров, А.С. Болдарев, С.В. Дьяченко, Е.Л. Карташева, О.Г. Ольховская, С.Н. Болдырев, И.В. Гасилова, В.А. Шмыров. Современные методы разработки программ для 3D-моделирования задач плазмодинамики (плазменной муль- 
тифизики). Вестник УГАТУ, Уфа, Уфимский Гос. Авиац. техн. унив., Сер. «Управление, вычисл. техника и информатика», 2011, т.15, No.5 (45), с.120-129;

V.A. Gasilov, G.A. Bagdasarov, A.S. Boldarev, S.V. D'yachenko, E.L. Kartasheva, O.G. Ol'hovskaya, S.N. Boldyrev, I.V. Gasilova, V.A. SHmyrov. Sovremennye metody razrabotki programm dlya 3D-modelirovaniya zadach plazmodinamiki (plazmennoj mul'tifiziki). Vestnik UGATU, Ufa, Ufimskij Gos. Aviacionnyj tekhnicheskij universitet, Ser. «Upravlenie, vychislitel'naya tekhnika, i informatika», 2011, t.15, No.5 (45), s.120-129.

12. В.Б. Вершинин, В.И. Делов, В.Н. Софронов. Разработка и реализация в комплексе Д численного метода расчета трехмерных нестационарных упругопластических течений // ВАНТ, сер. Математическое моделирование физических процессов, 2000, вып.3, с.9-24. V.B. Vershinin, V.I. Delov, V.N. Sofronov. Razrabotka i realizatsiia v komplekse D chislennogo metoda rascheta trekhmernykh nestatsionarnykh uprugoplasticheskikh techenii // VANT, ser. Matematicheskoe modelirovanie fizicheskih protsessov, 2000, vyp.3, s.9-24.

13. В.3. Партон, Е.М. Морозов. Механика упругопластического разрушения. - М.: Наука, 1981.

V.Z. Parton, E.M. Morozov. Mekhanika uprugoplasticheskogo razrusheniia. - M.: Nauka, 1981.

Поступила в редакцию 05.09 .2019

После доработки 05.09.2019

Принята к публикации 21.10.2019 